\title{
Efficiency optimization in a correlation ratchet with asymmetric unbiased fluctuations
}

\author{
Bao-Quan Ai ${ }^{1}$, Xian-Ju Wang ${ }^{1}$, Guo-Tao Liu ${ }^{1}$, De-Hua \\ $\mathrm{Wen}^{1,2}$, Hui-Zhang $\mathrm{Xie}^{2}$, Wei $\mathrm{Chen}^{3}$, and Liang-Gang Liu ${ }^{1}$ \\ ${ }^{1}$ Department of Physics, ZhongShan University, GuangZhou, China \\ 2 Department of Physics, South China University of technology, GuangZhou, China \\ 3 Department of Physics, JiNan University, GuangZhou, China
}

(Dated: October 28, 2018)

\begin{abstract}
The efficiency of a Brownian particle moving in periodic potential in the presence of asymmetric unbiased fluctuations is investigated. We found that there is a regime where the efficiency can be a peaked function of temperature, which proves that thermal fluctuations facilitate the efficiency of energy transformation, contradicting the earlier findings (H. kamegawa et al. Phys. Rev. Lett. 80 (1998) 5251). It is also found that the mutual interplay between asymmetry of fluctuation and asymmetry of the potential may induce optimized efficiency at finite temperature. The ratchet is not most efficiency when it gives maximum current.
\end{abstract}




\section{INTRODUCTION}

Much of the interest in non-equilibrium induced transport processes in concentrated on stochastically driven rachet 1] 2] 3]. This subject was motivated by the challenge to explain unidirectional transport in biological systems, as well as their potential novel technological applications ranging from classical non-equilibrium models [4] [5] to quantum systems 6] 7] 8]. Several models have been proposed to describe muscle's contraction [9][10] 11], or the asymmetric polymerization of actin filaments responsible of cell motility 1] [12].

Rectification of noise leading to unidirectional motion in ratchet systems has been an active field of research over the last decade. In these systems directed Brownian motion of particles is induced by nonequilibrium noise in the absence of any net macroscopic forces and potential gradients. Several physical models have been proposed: rocking ratchets 13], fashing ratchets [14], diffusion ratchets [15], correlation ratchets [16], etc. In all these studies the potential is taken to be asymmetric in space. It has also been shown that one can obtain unidirectional current in the presence of spatially asymmetric potentials. For these nonequilibrium systems external random force should be time asymmetric or the presence of space dependent mobility is required.

The energetics of these systems, which rectify the zero-mean fluctuations, are investigated in recent years [17] [18] [19]. To define optimal models for such ratchet systems, the maximization of the efficiency of energy transformation is inevitable. Much of interest was motivated by the elegant piece of work done by Magnasco [13], which showed that a Brownian particle, subject to external fluctuations, can undergo a non-zero drift while moving under the influence of an asymmetric potential. The temperature dependence of the current has been studied and it has been shown that the current can be a peaked function of temperature. He claimed that there is a region where the efficiency can be optimized at finite temperatures and the existence of thermal fluctuations facilitate the efficiency of energy transformation. Based on energetic analysis of the same model Kamegawa et al. 19] made a important conclusion that the efficiency of energy transformation cannot be optimized at finite temperatures and that the thermal fluctuations does not facilitate it. Recently, investigation of Dan et al. [17] showed that the efficiency can be optimized at finite temperatures in inhomogeneous systems with spatially varying friction coefficient in an adiabatically rocked rachet, and efficiency optimization in homogeneous nonadiabatical 
rachet systems was observed by Sumithra et al.[18]. The equation of whether the thermal fluctuations actually facilitate the energy transformation in forced homogeneous adiabatical ratchet systems is still unknown and it is the subject of the current investigation.

\section{THE MODEL}

We consider a rocking ratchet system subject to an external load:

$$
\frac{d x}{d t}=-\frac{\partial V_{0}(x)}{\partial x}-\frac{\partial V_{L}(x)}{\partial x}+F(t)+\sqrt{2 k_{B} T} \xi(t)
$$

where $x$ represents the state of the ratchet. $V_{0}$ is a periodic potential, $\xi(t)$ is a randomly fluctuating Gaussian white noise with zero mean and with autocorrelation function $<$ $\xi(t) \xi(s)>=\delta(t-s)$. Here $<\ldots>$ denotes an ensemble average over the distribution of the fluctuating forces $\xi(t)$. $V_{L}$ is a potential against which the work is done and $\frac{\partial V_{L}}{\partial x}=L>0$ is the load force. The geometry of potential $V(x)=V_{0}(x)+V_{L}(x)$ is displayed in Fig. 1a. $F(t)$ is some external driving force which is shown in Fig.1b. The evolution of the probability density for $x$ is given by the associated Fokker-Planck equation,

$$
\frac{\partial P(x, t)}{\partial t}=\frac{\partial}{\partial x}\left[k_{B} T \frac{\partial P(x, t)}{\partial x}+\left(V^{\prime}(x)-F(t)\right) P(x, t)\right]=-\frac{\partial j}{\partial x} .
$$

If $F(t)$ changes very slowly, there exists a quasi-stationary state. In this case, the average current of the particle can be solved by evaluating the constants of integration under the normalization condition and the periodicity condition of $P(x)$, and the current can be obtained and expressed as [13]

$$
j(F(t))=\frac{P_{2}^{2} \sinh \left[\lambda(F(t)-L) / 2 k_{B} T\right]}{k_{B} T(\lambda / Q)^{2} P_{3}-(\lambda / Q) P_{1} P_{2} \sinh \left[\lambda(F(t)-L) / 2 k_{B} T\right]},
$$

where

$$
\begin{gathered}
P_{1}=\Delta+\frac{\lambda^{2}-\Delta^{2}}{4} \frac{F(t)-L}{Q}, \\
P_{2}=\left(1-\frac{\Delta(F(t)-L)}{2 Q}\right)^{2}-\left(\frac{\lambda(F(t)-L)}{2 Q}\right)^{2}, \\
P_{3}=\cosh \left\{[Q-0.5 \Delta(F(t)-L)] / k_{B} T\right\}-\cosh \left[\lambda(F(t)-L) / 2 k_{B} T\right],
\end{gathered}
$$

where $\lambda=\lambda_{1}+\lambda_{2}$ and $\Delta=\lambda_{1}-\lambda_{2}$. The average current, the quantity of primary interest, is given by

$$
J=\frac{1}{\tau} \int_{0}^{\tau} j(F(t)) d t
$$




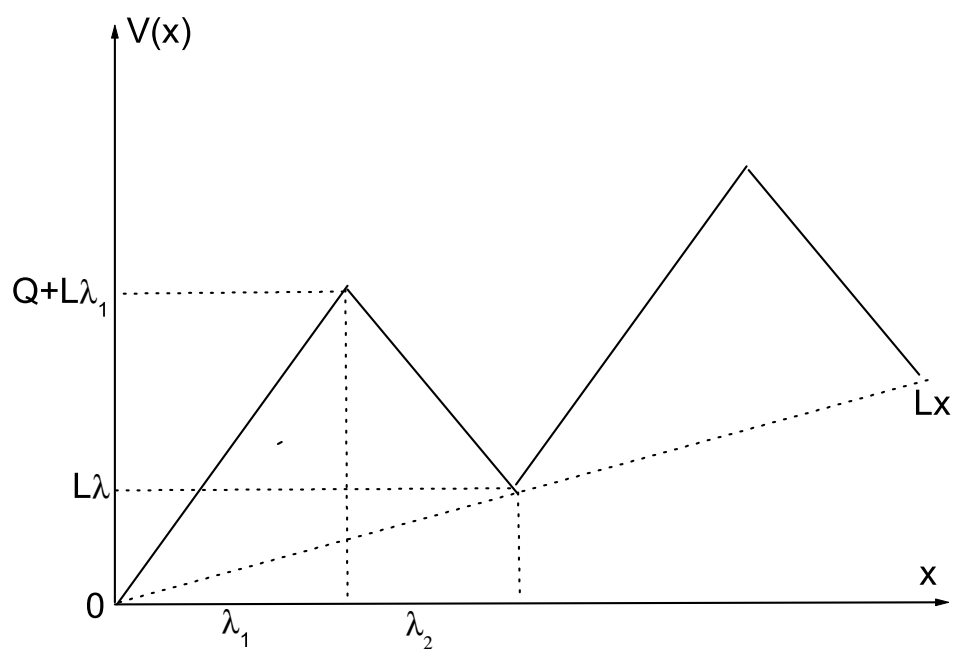

(b)

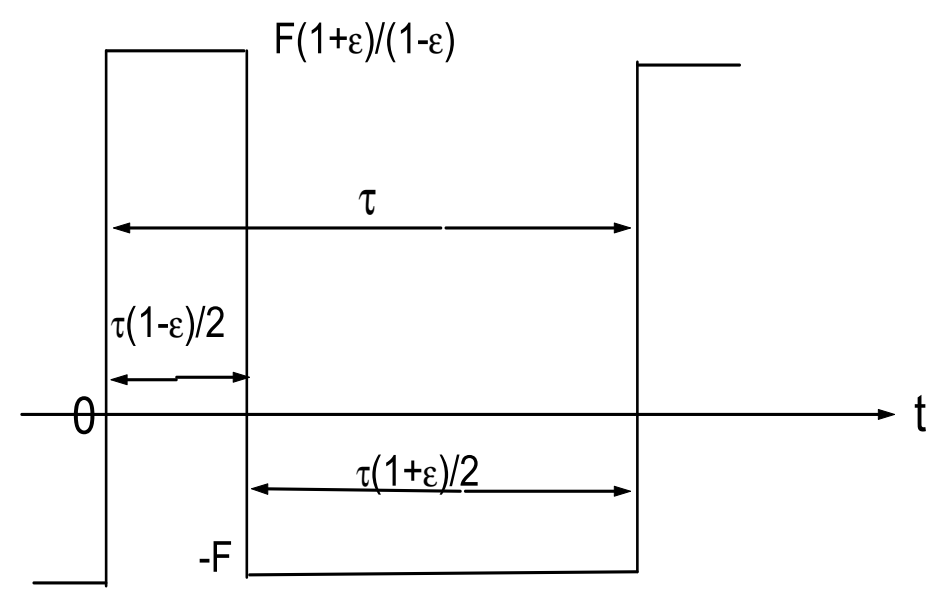

FIG. 1: (a) Schematic illustration of the potential, $V(x)=V_{0}(x)+V_{L}(x), V_{0}(x)$ is a piecewise linear and periodic potential. $V_{L}$ is a potential due to the load. The period of the potential is $\lambda=\lambda_{1}+\lambda_{2}$, and $\Delta=\lambda_{1}-\lambda_{2}$. (b) The driving force $F(t)$ which preserved the zero mean $\langle F(t)\rangle=0$, where the temporal asymmetry is given by the parameter $\varepsilon$. 
where $\tau$ is the period of the driving force $F(t)$, which is assumed longer than any other time scale of the system in this adiabatic limit. Magnasco considered this case, but only for $F(t)$ symmetric in time. Here will again consider a driving with a zero mean, $\langle F(t)\rangle=0$, but which is asymmetric in time [20]

$$
\begin{aligned}
F(t) & =\frac{1+\varepsilon}{1-\varepsilon} F,\left(n \tau \leq t<n \tau+\frac{1}{2} \tau(1-\varepsilon)\right), \\
& =-F,\left(n \tau+\frac{1}{2} \tau(1-\varepsilon)<t \leq(n+1) \tau\right) .
\end{aligned}
$$

In this case the time averaged current is easily calculated,

$$
J=\frac{1}{2}\left(j_{1}+j_{2}\right)
$$

where $j_{1}=(1-\varepsilon) j\left(\frac{1+\varepsilon}{1-\varepsilon} F\right)$ and $j_{2}=(1+\varepsilon) j(-F)$.

The input energy $R$ per unit time from external force to the ratchet and the work $W$ per unit time that the ratchet system extracts from the fluctuation into the work are given respectively [19]:

$$
\begin{aligned}
& R=\frac{1}{t_{j}-t_{i}} \int_{x\left(t_{i}\right)}^{x\left(t_{j}\right)} F(t) d x(t), \\
& W=\frac{1}{t_{j}-t_{i}} \int_{x\left(t_{i}\right)}^{x\left(t_{j}\right)} d V(x(t)) .
\end{aligned}
$$

For the square wave, they yield:

$$
\begin{aligned}
& <R>=\frac{1}{2} F\left(j_{1}-j_{2}\right), \\
& <W>=\frac{1}{2} L\left(j_{1}+j_{2}\right) .
\end{aligned}
$$

Thus the efficiency $\eta$ of the system to transform the external fluctuation to useful work is given by

$$
\eta=\frac{<W>}{<R>}=\frac{L\left(j_{1}+j_{2}\right)}{F\left(j_{1}-j_{2}\right)} .
$$

which in turn, being $\frac{j_{2}}{j_{1}}<0$, can be written as

$$
\eta=\frac{L}{F}\left(\frac{1-\left|j_{2} / j_{1}\right|}{1+\left|j_{2} / j_{1}\right|}\right)
$$




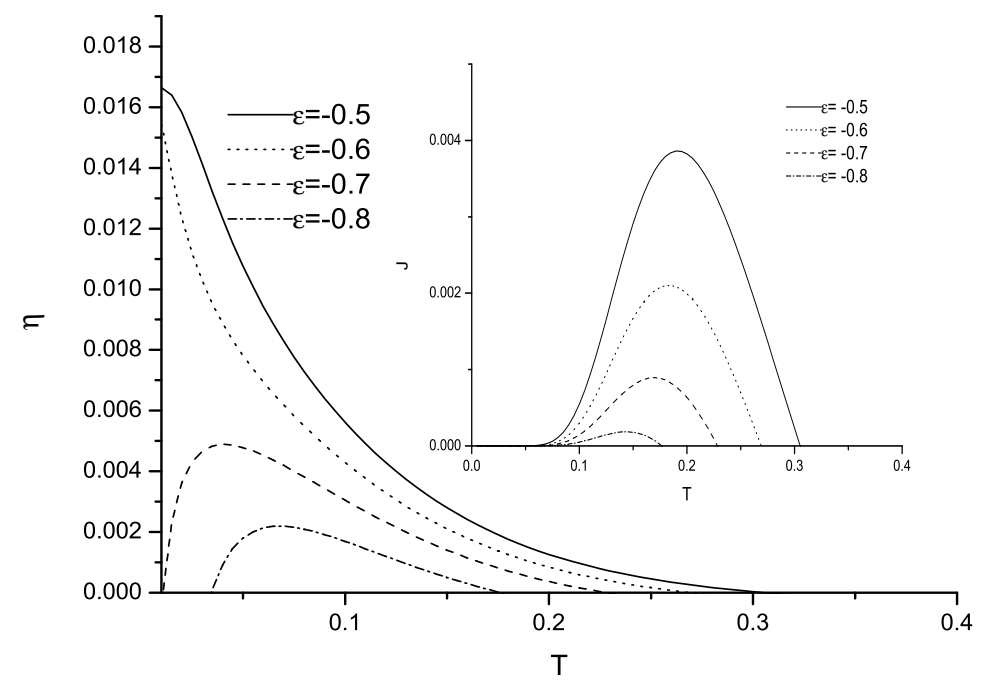

FIG. 2: Efficiency $\eta$ as a function of temperature $T$ for different values of asymmetric parameters $\varepsilon=-0.5,-0.6,-0.7,-0.8, F=1.0, \lambda=1.0, \Delta=0.7, Q=1.0$ and $L=0.01$. The inset shows the net current $J$ as function of $T$ for the same parameters.

\section{RESULTS AND DISCUSSION}

we have calculated the efficiency and the net current as a function of temperature $T$ for the case where asymmetric unbiased fluctuations are applied, and the results are shown in Fig. 2- Fig. 6.

In Fig. 2 we plot the efficiency $\eta$ as a function of the temperature for different values of $\varepsilon$ $(\varepsilon<0)$ with the parameter values, $F=1.0, \lambda=1.0, \Delta=0.7, Q=1.0$ and $L=0.01$. From the figure we can see that the efficiency is a decreasing function of temperature for the cases of $\varepsilon=-0.5$ and $\varepsilon=-0.6$, which shows that the presence of thermal fluctuation dose not help efficient energy transformation by ratchet. But for the cases of $\varepsilon=-0.7$ and $\varepsilon=-0.8$ the efficiency can be optimized at finite temperatures. In contradiction with the results of Ref (19) we found a region where the efficiency attains a maximum at a finite temperature. This shows that thermal fluctuations may facilitate the energy conversion for asymmetric unbiased fluctuations. The current is a peaked function of temperature for corresponding parameters as shown in the inset. The highest temperature of the ratchet decreases with the value of the asymmetric parameters $\varepsilon$ of fluctuations and the lowest temperature of 
the ratchet does not change with the $\varepsilon$, which indicates that the asymmetric parameters are sensitive to the highest working temperature of the ratchet. The peak shift to lower temperature region with decreasing value of the asymmetric parameters $\varepsilon$. Comparing Fig. 2 with the inset we can see that the temperature corresponding to maximum current is not the same as the temperature at which the efficiency is maximum, which is consistent with the previous results [17] 18] 19].

From Eq. (16) we can know that the efficiency $\eta$ depends on the ratio $\left|\frac{j_{2}}{j_{1}}\right|$. If the function is monotonically increasing, $\eta$ should be a monotonically decreasing function of the temperature. In Fig. 3 we plot the fluxes obtained for the case of $\varepsilon=-0.5$ (shown in

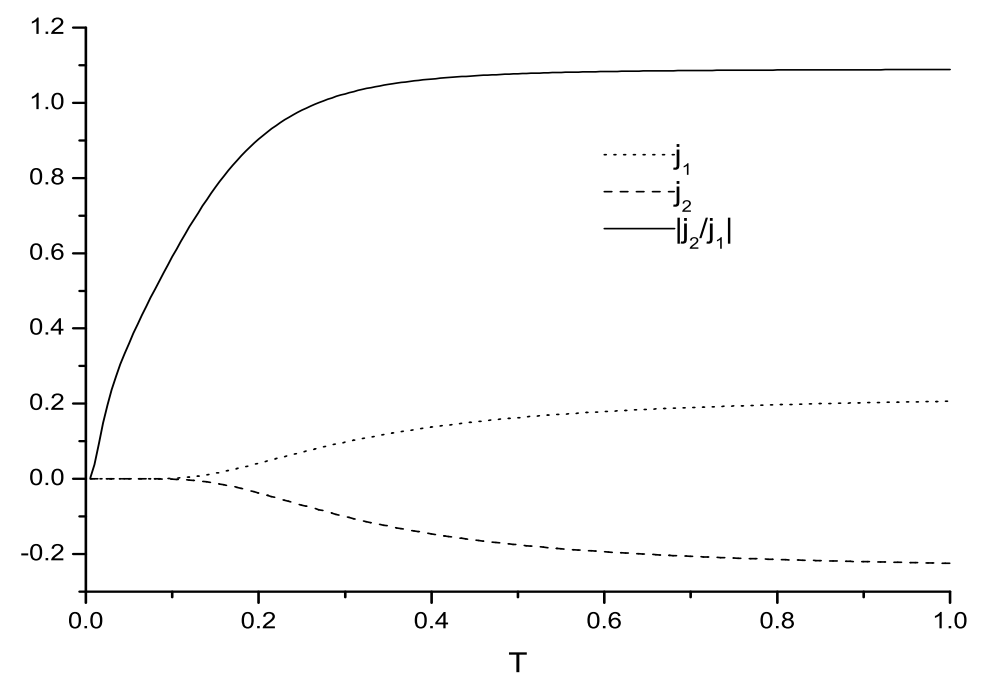

FIG. 3: Plot of currents $j_{1}, j_{2}$ and $\left|\frac{j_{2}}{j_{1}}\right|$. The condition is the same as the case $\varepsilon=-0.5$ in Fig. 2

Fig. 2). From Fig. 3 we can see that the ratio $\left|\frac{j_{2}}{j_{1}}\right|$ is a monotonically increasing function of temperature, which indicates that the efficiency $\eta$ is decreasing function of temperature. However, for the case of $\varepsilon=-0.7$ (see Fig. 4) the ratio $\left|\frac{j_{2}}{j_{1}}\right|$ displays a clear minimum at the same value of the temperature which corresponds to maximum of $\eta$ in Fig. 2.

In Fig. 5 we plot the efficiency $\eta$ as a function of the temperature $T$ for different values of slope degree of potenial $\Delta(\Delta<0)$ with the parameter values, $F=1.0, \lambda=1.0, \varepsilon=0.7$, $Q=1.0$ and $L=0.01$. From the figure, we can see that with decreasing of $\Delta$ the efficiency function of temperature becomes from a monotonically decreasing function to a peaked function. This shows that the thermal fluctuations actually facilitate the energy transformation 


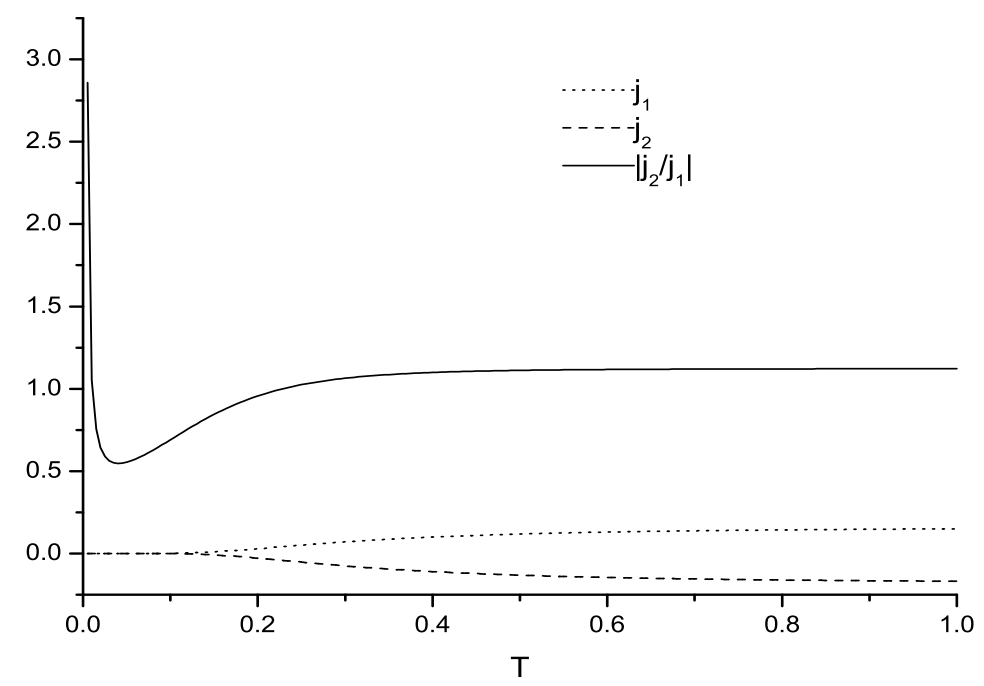

FIG. 4: Plot of currents $j_{1}, j_{2}$ and $\left|\frac{j_{2}}{j_{1}}\right|$. The condition is the same as the case $\varepsilon=-0.7$ in Fig. 2

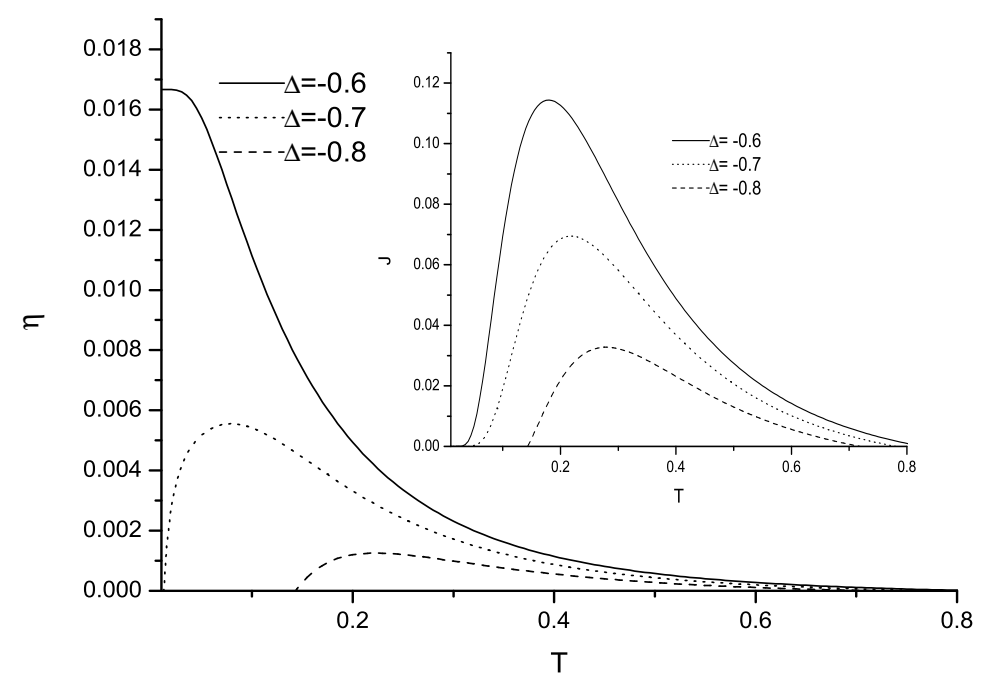

FIG. 5: Efficiency $\eta$ as a function of temperature $T$ for different values of asymmetric parameters $\Delta=-0.6,-0.7,-0.8, F=1.0, \lambda=1.0, \varepsilon=0.7, Q=1.0$ and $L=0.01$. The inset shows the net current $J$ as function of $T$ for the same parameters. 
in some region. The corresponding current is a peaked function of temperature for the same parameters as shown in the inset. The height of the peak decreases with the value of $\Delta$. The lowest temperature of the ratchet changes with $\Delta$ drastically while the highest temperature of the ratchet does not change with the $\Delta$ and the peak shift to higher temperature region with decreasing value of $\Delta$, which is opposite to the inset of the Fig. 2.

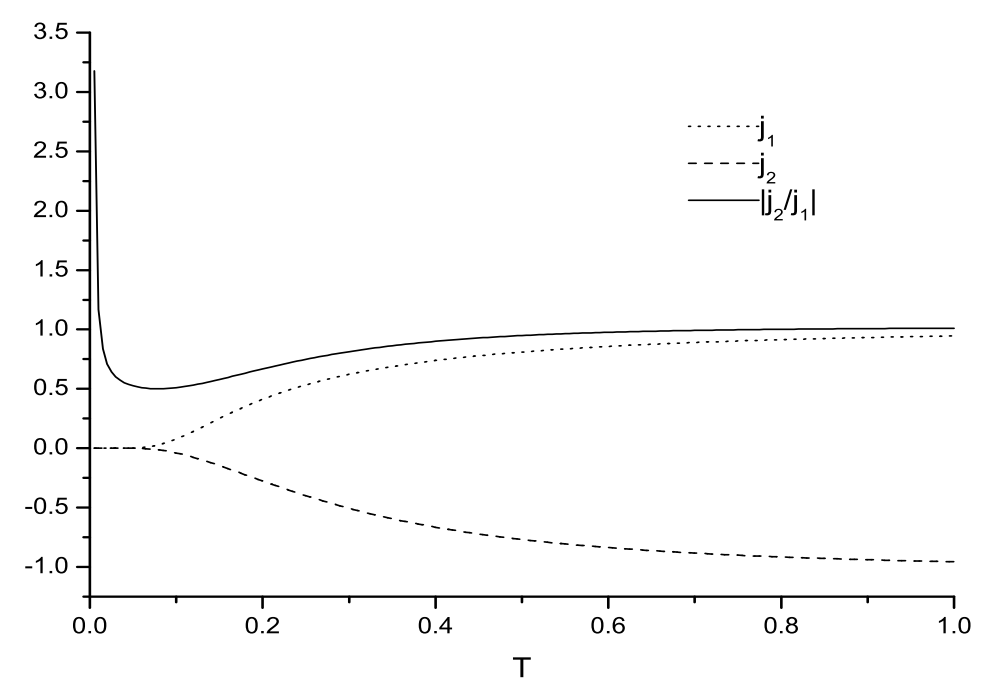

FIG. 6: Plot of currents $j_{1}, j_{2}$ and $\left|\frac{j_{2}}{j_{1}}\right|$. The condition is the same as the case $\Delta=-0.7$ in Fig. 5

In Fig. 6, we plot the fluxes of the temperature for the case of $\Delta=-0.7$ (shown in Fig. 5). From the Fig. 6 we can see that the ratio $\left|\frac{j_{2}}{j_{1}}\right|$ displays a minimum at the same value of the temperature, which corresponds to maximum of $\eta$ in Fig. 5

\section{CONCLUSION}

In present paper, the transport of a Brownian particle moving in spatially asymmetric potential in the presence of asymmetric unbiased fluctuations is investigated. In contradiction with the previous findings, our results show that the mutual interplay between asymmetry of fluctuation and asymmetry of potential may cause an optimized efficiency of energy conversion. This proves the claim made by Magnasco that there is a region of the operating 
regime where the efficiency can be optimized at finite temperatures. The temperature corresponding to maximum current is not the same as the temperature at which the efficiency is maximum. The asymmetry $\varepsilon$ of fluctuation is sensitive to the high temperature working region of the ratchet while the asymmetry $\Delta$ of potential affects the low temperature working region drastically.

In this paper, the main features introduced by the temporal asymmetry are the interplay of lower potential barriers in positive direction relative to negative direction and the corresponding shorter and longer times respectively the force is felt. These type of competitive effects appear ubiquitously in systems [20] where there is an interplay between thermal activation and dynamics.

The project supported by National Natural Science Foundation of China (Grant No. of 10275099) and GuangDong Provincial Natural Science Foundation (Grant No. of 021707 and 001182).

[1] F. Julicher, A. Adjari, J. Prost, Rev. Mod. Phys. 69 (1997) 1269.

[2] B. Q. Ai, X. J. Wang, G. T. Liu and L. G. Liu, Phys. Rev. E 67 (2003) 022903.

[3] P. Reimann, C. Van den Broeck, H. Linke, P. Hanggi,etc., Phys. Rev. Lett. 87 (2001) 010602.

[4] J. Rousselet, L. Salome, A. Adjari and J. Prost, Nature 370 (1994) 446.

[5] L. P. Faucheux et al., Phys. Rev. Lett. 74 (1995) 1504.

[6] I. Derenyi, C. Lee, A. L. Barabasi, Phys. Rev. Lett. 80 (1998) 1473.

[7] C. Mennerat-Robilliard et al., Phys. Rev. Lett. 82 (1999) 851.

[8] C. S. Lee et al., Nature 400 (1999) 337.

[9] R. D. Astumian, M. Bier, Phys. Rev. Lett. 72 (1994) 1766.

[10] B. Q. Ai, X. J. Wang, L. G. Liu, M. Nakano and H. Matsuura, Chin. Phys. Lett.19 (2002) 137.

[11] B. Q. Ai, X. J. Wang, L. G. Liu, M. Nakano and H. Matsuura, Commun. Theor. Phys.37 (2002) 125.

[12] B. Q. Ai, X. J. Wang, L. G. Liu, M. Nakano and H. Matsuura, Information, VOL.6 (2003)187.

[13] M. O. Magnasco, Phys. Rev. Lett. 71 (1993) 1477.

[14] P. Hanggi and R. Bartussek, Nonlinear physics of complex system - Current status and Future Trends, 476, Spring, Berlin, (1996), 294.

[15] P. Reimann, R. Bartussek, R. Haussler and P. Hanggi, Phys. Lett. A 215 (1994) 26.

[16] C. R. Doering, W. Horsthemke and J. Riordan, Phys. Rev. Lett. 72 (1994) 2984.

[17] D. Dan, M. C. Mahato and A. M. Jayannavar, Phys. Rev. E 63 (2001) 056307.

[18] K. Sumithra and T. Sintes, Physica A 297 (2001) 1.

[19] H. Kamgawa, T. Hondou and F. Takagi, Phys. Rev. Lett. 80 (1998) 5251.

[20] D. R. Chialvo and M. M. Millonas, Phys. Lett. A 209 (1995) 26. 